\title{
Patientensicherheit in der Anästhesie Kann der Anästhesist das Outcome verbessern?
}

Jochen Renner • Matthias Grünewald • Berthold Bein

Trotz fachspezifischer pharmakologischer und technologischer Innovationen wissen wir grundsätzlich, dass in unserem Fachgebiet perioperativ Änderungen im physiologischen Gleichgewicht induziert werden, die das Risiko postoperativer Komplikationen und damit konsequenterweise auch das Mortalitätsrisiko erhöhen können. Erfreulicherweise hat der Aspekt „Patientensicherheit in der Anästhesie“ in den letzten Jahren enorm an Aufmerksamkeit gewonnen und es gibt mittlerweile vielversprechende Konzepte, die nachweislich die Patientensicherheit verbessern können.

Perioperative Patientensicherheit „This declaration emphasises the key role of anaesthesiology in promoting safe perioperative care." Dieser Satz findet sich am Ende der Deklaration von Helsinki zur Patientensicherheit in der Anästhesiologie, die am 12. Juni 2010 von führenden Repräsentanten der anästhesiologischen Fachgesellschaften in Europa unterzeichnet wurde [1].

Auch wenn die Anästhesiologie in den letzten Jahrzehnten eine Schlüsselrolle bei der Optimierung der perioperativen Patientensicherheit eingenommen hat, wäre es die falsche Schlussfolgerung, sich auf diesem Erfolg nun auszuruhen. Die nationalen Fachgesellschaften können auf eine Vielzahl von Innovationen zurückblicken, die in den letzten 60 Jahren nach Einführung des Facharztes für Anästhesie die Patientensicherheit nachhaltig verbessert haben. Dennoch müssen wir uns auch in den kommenden Jahren den demografischen Herausforderungen stellen - insbesondere in Kombination mit den technologischen Innovationen der operativen Fachrichtungen - und unser perioperatives Management adjustieren. Mit diesem ambitionierten Ansatz wäre die Frage, ob wir uns als Anästhesisten auf die bestmögliche intraoperative Versorgung unserer Patienten beschränken oder ob wir im interdisziplinären Ansatz das perioperative Outcome verbessern wollen, klar beantwortet.

Definition "Outcome" Der fachübergreifende Begriff „Outcome“ hat durchaus auch im deutschsprachigen Bereich seinen Stellenwert gefunden und wird im Wesentlichen ohne weitere klare
Definition als das Ergebnis einer medizinischen Intervention verstanden. Somit beschreibt „Outcome“ zunächst die Effektivität eines Behandlungskonzepts und die idealerweise damit verbundene Reduktion von Morbidität und Mortalität, die Erhaltung oder die Wiederherstellung der Leistungsfähigkeit eines Patienten [2]. In diesem Sinne wird der Begriff „Outcome“, der durchaus deutlich komplexer behandelt und verstanden werden könnte, auch im Folgenden verwendet.

\section{Historie \\ $\nabla$}

Die Anfänge Vor etwas mehr als 150 Jahren führte der Zahnarzt William T. Green Morton am 16. Oktober 1846 die erste erfolgreiche Äthernarkose im Operationshörsaal des Massachusetts General Hospital in Boston durch [3]. Bis zu diesem Zeitpunkt bleibt im historischen Rückblick bezüglich der Stress- und Schmerzlinderung bei Operationen festzuhalten: Die Chirurgie war barbarisch, die Anästhesie primitiv und die postoperative Sterblichkeit wohl eher im hohen zweistelligen Prozentbereich angesiedelt.

Eine bahnbrechende Innovation im Sinne eines gesicherten Atemwegs, die Intubationsnarkose, ist dem Heidelberger Chirurgen Franz Kuhn zuzuschreiben. Allerdings gab es damals (1905) erhebliche Vorbehalte seiner chirurgischen Kollegen, insbesondere durch Ferdinand Sauerbruch. Das Outcome-relevante Potenzial dieser Innovation sollte erst viele Jahre später Beachtung finden.

Wachsende Anerkennung Im weiteren Verlauf des 20. Jahrhunderts wurden die Bemühungen, das Fachgebiet Anästhesie intensiver zu erforschen, rasant durch entsprechende Erfolge belohnt. Unterschiedliche Narkoseverfahren wurden entwickelt und schnell war sich die Mehrheit der Chirurgen sicher:

„Ein Spezialismus ist günstig für jede Art der Narkose. Ich bin überzeugt, dass wir keinen Fortschritt in der praktischen Narkosekunst machen werden, wenn wir in der Zukunft nur Schwestern und Gele- 
genheitsnarkotiseuren die Narkosen überlassen, die ebenso gefährlich sind wie die Gelegenheitsoperateure. Nicht die Narkosewissenschaft liegt im Argen, sondern die Narkosekunst bedarf der Neuerung“. (Zitat Helmut Schmidt, Hamburger Chirurg) [4]

Bedeutung für Patientensicherheit Im Jahr 1949 verdeutlichte der erste Lehrstuhlinhaber für Anästhesie in Europa, Sir Robert Macintosh, mit einer Aussage sehr anschaulich, wie schmal der Grad zwischen Sicherheit und desaströsem Outcome in der Anästhesie sein kann:

•,Deadly easy - easily dead“.

Darüber hinaus warb er als einer der Ersten für den Einsatz hochqualifizierten Anästhesiepersonals, um die Anästhesie-bedingte Mortalität minimieren zu können [5]: „There should be no deaths due to anaesthesia“. In der Originalarbeit von Macintosh aus dem Jahr 1949 werden verschiedene klinisch-anästhesiologische Szenarien beschrieben, die mittel- oder unmittelbar zum Tod der Patienten geführt haben. Hier schon wird deutlich, dass generell $\mathrm{zu}$ unterscheiden ist zwischen Anästhesie-bedingter und Anästhesieassoziierter Morbidität und Mortalität.

\section{Einfluss der Anästhesie auf Morbidität und Mortalität}

Schwer zu messen Wie lassen sich heute Sicherheit und Effektivität der anästhesiologischen Patientenversorgung am ehesten messen? Üblicherweise geschieht dies anhand definierter Endpunkte wie Morbidität und Mortalität sowie der Anzahl unerwünschter Ereignisse. Nun sind Morbidität und Mortalität nicht zwangsläufig mit Anästhesie assoziiert und lassen sich häufig nur schwer einzelnen der perioperativen Phasen zuordnen. Darüber hinaus scheitert die Objektivierung sog. unerwünschter Zwischenfälle an einer nicht flächendeckend vorhandenen Bereitschaft diese auch tatsächlich zu dokumentieren [6] Dennoch wissen wir grundsätzlich sehr wohl, dass die Anästhesiologie das Potenzial hat, Änderungen im physiologischen Gleichgewicht zu induzieren, die durchaus das Risiko postoperativer Komplikationen und konsequenterweise auch das Mortalitätsrisiko erhöhen können.

\section{Anästhesie-bedingte Mortalität}

$\nabla$

Deutliche Reduktion Die Anästhesie-bedingte Mortalität beschreibt die unmittelbare Kausalität einer anästhesiologischen Maßnahme mit dem Versterben eines Patienten. In dieser Hinsicht können wir unseren Patienten heute im Vergleich zu 1940 sehr viel mehr Sicherheit bieten ( $\bullet$ Abb. 1). Ist man damals noch von einer Anästhesie-bedingten Mortalität von 10 pro 10000

\section{Entwicklung der Anästhesie-bedingten Mortalität}

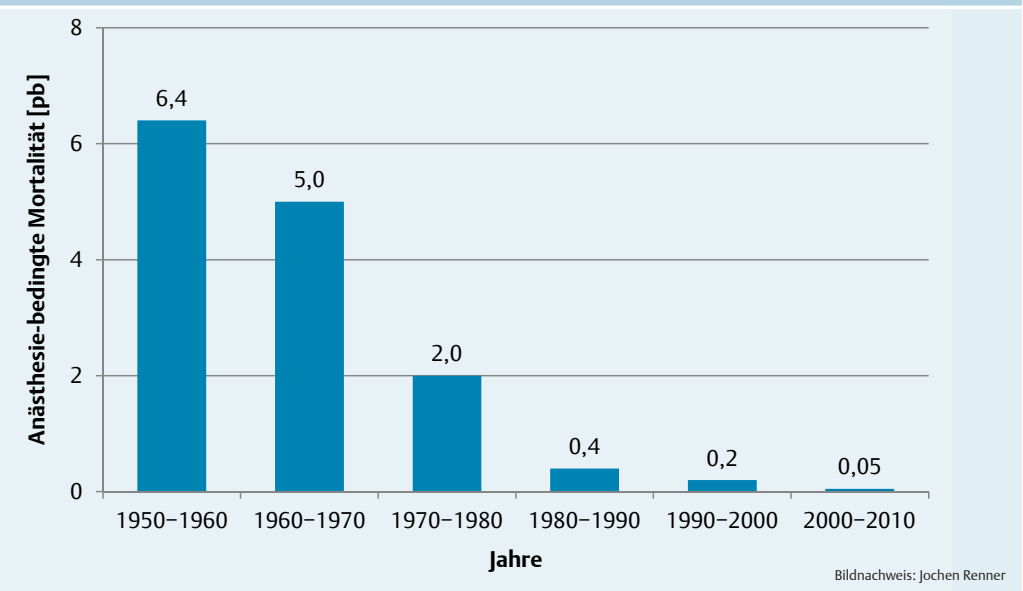

Abb. 1 Entwicklung der Anästhesie-bedingten Mortalität pro 10000 Anästhesien in den letzten 60 Jahren. Daten aus [7, 10, 47-52].

Anästhesien ausgegangen, liegen wir heute zwischen 0,055-0,1 pro 10000 (je nach Quelle), mit einem deutlich höheren Anteil in den Entwicklungsländern [7-10].

Schon in den 1980er-Jahren konnte eine sprunghafte Reduktion der Anästhesie-bedingten Mortalität beobachtet werden, die zeitlich durchaus parallel zur Entwicklung und Implementierung weiterer Sicherheitsstandards wie der Pulsoxymetrie und der Kapnometrie zu sehen ist [11]. Dieser Zusammenhang wurde bislang nicht unmittelbar in großen randomisierten Studien nachgewiesen und wird auch aus ethischen Gründen in Zukunft nicht mehr Gegenstand wissenschaftlicher Untersuchungen werden.

$\mathrm{Zu}$ dem Thema liegt eine Reihe interessanter, meist retrospektiver Untersuchungen aus verschiedenen Kontinenten vor. Deren Daten sind jedoch aufgrund der stark differierenden Methodik der Untersuchungen sehr schwer zu interpretieren und zu übertragen.

Die Anästhesie-bedingte Mortalität konnte in den letzten Jahrzehnten deutlich reduziert werden und liegt derzeit zwischen 0,055-0,1 Todesfällen pro 10000 Anästhesien.

\section{Anästhesie-assoziierte Mortalität \\ $\nabla$}

Definition Der Begriff der Anästhesie-assoziierten Mortalität ist deutlich weiter gefasst. Er beschreibt die Mortalität im Rahmen einer Operation/Intervention, die unter einer Form der Anästhesie durchgeführt wurde (Allgemein- oder Regionalanästhesie). Eine unmittelbare Kausalität zwischen der anästhesiologischen Maßnahme und dem Versterben kann nicht zwangsläufig hergestellt werden. Konsequenterweise ist der exakte Stellenwert der Anästhesie nur schwer zu ermitteln. 
Arbous et al. Arbous und Mitarbeiter aus den Niederlanden haben sich mit der Frage beschäftigt, inwieweit sich Anästhesie-assoziierte Risikofaktoren identifizieren lassen, die in Zusammenhang mit der Entwicklung schwerer Komplikationen (definiert als Koma und Tod) innerhalb der ersten $24 \mathrm{~h}$ postoperativ stehen [7]. Hierzu wurde zwischen 1995 und 1997 eine Kohorte von 869483 Patienten retrospektiv untersucht. Die Inzidenz von postoperativem Tod innerhalb der ersten $24 \mathrm{~h}$ lag bei 8,8 pro 10000 Anästhesien, die Inzidenz von postoperativem Koma bei 0,5 pro 10000. Darüber hinaus konnten die Autoren Faktoren ermitteln, die das Risiko, eine dieser schwerwiegenden Komplikation zu erleiden, relevant reduzierten:

- Equipment-Check mit Checkliste und Dokumentation

- direkt verfügbarer Anästhesist im OP-Saal

- kein Wechsel des Anästhesisten während der Narkose

- postoperative Schmerztherapie (insbesondere Epiduralanästhesie)

- Antagonisierung der Muskelrelaxanzien und/ oder der Opioide

- Präsenz einer Anästhesieassistenz im OP

Li et al. Eine Arbeitsgruppe um Li und Mitarbeiter aus den Vereinigten Staaten wertete in einer aufwendigen retrospektiven Analyse einer ICD10-Kodierungs-Datenbank, die auf anästhesiologische Komplikationen spezifiziert war, Todesbescheinigungen aus den Jahren 1999-2005 in den USA aus [12]. In dieser Arbeit zeigte sich eine Anästhesie-assoziierte Mortalitätsrate von 8,2 pro 1 Mio. chirurgischer Eingriffe unter Anästhesie $(0,082$ pro 10000 Anästhesien) mit der höchsten Sterblichkeit bei den > 85-jährigen Patienten.

Lienhart et al. In einer Untersuchung aus Frankreich stellten Lienhart und Kollegen aus $>500000$ Todesbescheinigungen letztendlich in 4200 Fällen ein möglicher Bezug zu Anästhesien fest und erfragten und überprüften später Anzahl und Charakteristika der dokumentierten Todesfälle [10]. Die Autoren ermittelten

- eine Anästhesie-bedingte Mortalität von 0,069 pro 10000 Anästhesien und

- eine Anästhesie-assoziierte Mortalität von 0,47 pro 10000 Anästhesien.

Darüber hinaus konnten sie einen deutlichen Zusammenhang zwischen der ASA-Klassifikation und der Mortalität sowie dem Alter und der Mortalität zeigen ( $\odot$ Abb. 2 und 3). $42 \%$ der Todesfälle wurden in den ersten $24 \mathrm{~h}$ postoperativ beobachtet. Hiervon waren

- 46,6\% mit einer Überdosierung von Anästhetika assoziiert,

-42,5\% beruhten auf Nebenwirkungen von in therapeutischer Dosierung verabreichten Anästhetika und
- 3,6\% der Todesfälle standen in Zusammenhang mit Schwangerschaft und Geburtshilfe.

Als Prädiktoren für ein erhöhtes Risiko wurden Hypotension, Anämie und postoperative ischämische Ereignisse genannt.

Monk et al. Auch andere Untersuchungen haben sich Anästhesie-spezifischen, im Wesentlichen intraoperativen Prädiktoren eines erhöhten Risikos für schwere postoperative Komplikationen gewidmet. So konnten Monk und Kollegen in einer prospektiven Kohorten-Studie aus dem Jahr 2005 an 1064 Patienten zu großen abdominalchirurgischen Eingriffen zeigen, das 5,5\% der Patienten innerhalb eines Jahres verstarben und 10,3\% der Verstorbenen > 65 Jahre alt waren [13]. Auch in dieser Arbeit konnten Prädiktoren klar benannt werden:

- Alter > 65 Jahre

- Komorbiditäten

- bispektraler Index (BIS) < 45 (Anzahl der Minuten mit einem BIS-Wert <45)

- Hypotonie (Anzahl der Minuten mit einem syst. Blutdruck $<80 \mathrm{mmHg}$ )

Weitere Prädiktoren In einer Untersuchung an 749 Patienten zu großen nicht herzchirurgischen Eingriffen wurden OP-Dauer, Tachykardie und Hypertonie als unabhängige Prädiktoren einer erhöhten Morbidität, Mortalität und einer verlängerten Krankenhausaufenthaltsdauer identifiziert [14]. Diese Liste lässt sich durch mehrere Studien und durch eine Reihe weiterer Prädiktoren für ein erhöhtes postoperatives Risiko für schwere Komplikationen ergänzen: Brady- und Tachykardie, Hypo- und Hypertonie, OP-Dauer, hohe Blutdruckvariation, pulmonale Hypertonie, Triple-low-Situation (= niedriger Blutdruck, niedriger Bispektralindex, niedrige min. alveoläre Konzentration [MAC]) [15-18]. Gemeinsames Merkmal dieser Prädiktoren ist die Tatsache, dass deren Erkennung (z.B. pulmonale Hypertonie), deren adäquate Überwachung und konsequenterweise deren Therapie in der Hand der Anästhesie, des betreuenden Anästhesisten liegen.

Die Angaben zur Anästhesie-assoziierten Mortalität sind nicht zuletzt aufgrund großer methodologischer Unterschiede der in erster Linie retrospektiven Analysen auch heute noch sehr inhomogen und liegen zwischen 0,08-5,5 (ASA-IV-Patienten) pro 10000 Narkosen.

\section{Was können wir tun, um das Outcome zu verbessern?}

Vielversprechende Ansätze Derzeit gibt es eine Reihe vielversprechender, weitgehend „evidenzbasierter“ Konzepte in der Anästhesiologie, für die gezeigt werden konnte, dass sie die Patienten- 


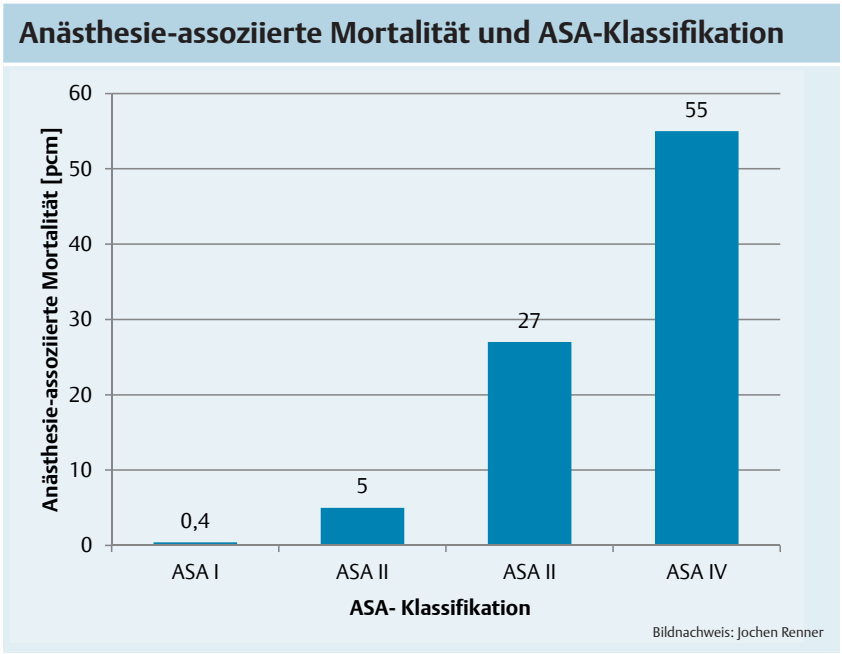

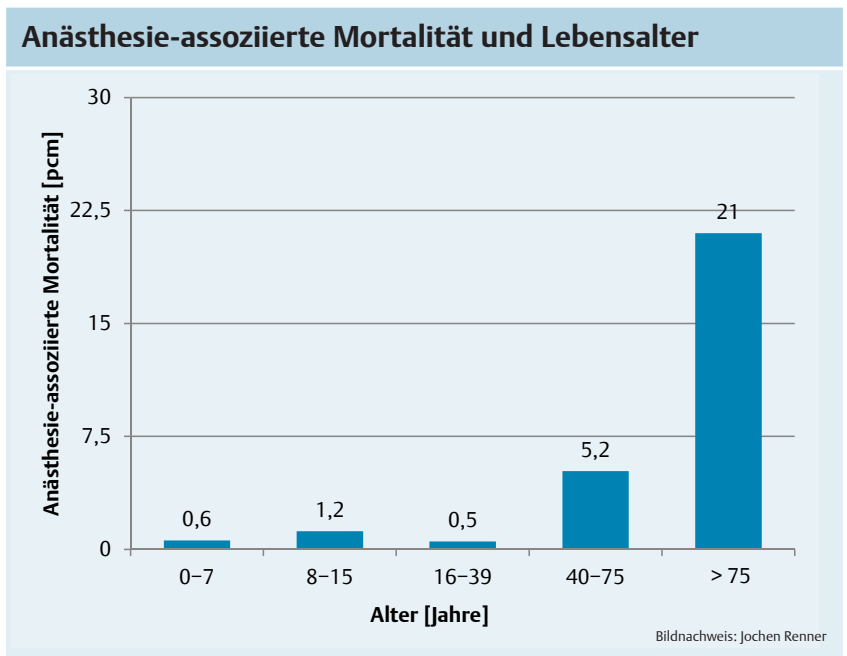

Abb. 2 (links) Anästhesieassoziierte Mortalität und ASAKlassifikation (ASA = American Society of Anesthesiologists) in Frankreich pro 100000 Anästhesien. Daten aus [10].

Abb. 3 (rechts) Anästhesieassoziierte Mortalität und Lebensalter in Frankreich pro 100000 Anästhesien. Daten aus [10].
„Surgical Safety Checklist“ Im Hinblick auf eine erhöhte Patientensicherheit hat die WHO im Jahr 2007 eine Checkliste („Surgical Safety Checklist“) für ein interdisziplinäres Briefing im OP-Saal entworfen. Grundlage dieses Briefings ist ein Zusammentreffen aller Teammitglieder zum Austausch und Update aller nötigen Informationen, um den bestmöglichen Ablauf der anstehenden Aufgabe gewährleisten zu können. Ein derartiges Vorgehen ist seit vielen Jahren im Rahmen der Flugsicherheit Vorschrift.

In einer weltweit ausgerichteten prospektiven Studie an Kliniken der Primär- und Maximalversorgung wurde die Surgical Safety Checklist evaluiert. Haynes und Mitarbeiter konnten zeigen, dass durch die Einhaltung der Checkliste

- die Mortalität bei großen Eingriffe signifikant von $1,5 \%$ auf $0,8 \%$ sowie

- schwere postoperative Komplikationen signifikant von $11 \%$ auf $7 \%$ reduziert werden konnten [19].

Aufgrund dieser Ergebnisse hat die WHO 2009 empfohlen, diese Checkliste bei allen Operationen anzuwenden - eine Empfehlung, die von der Mehrzahl anästhesiologischer und chirurgischer Fachgesellschaften übernommen wurde [20-22]

Vermeidung von Medikationsfehlern Neben verschiedenen anderen Aspekten wird in der eingangs schon erwähnten Deklaration von Helsinki zur Patientensicherheit auch die Bedeutung der Medikationssicherheit durch die Forderung nach klinisch sicher darstellbaren Standards und Strukturen zur Überprüfung der gängigen Medikamente anhand standardisierter farblicher Kennungen verdeutlicht. In diesem Kontext wurde von der Internationalen Organisation für Standar- disierung (ISO) die Norm ISO-26825 zur farblichen Kennzeichnung anästhesiologischer Medikamente entwickelt, die bereits in vielen Ländern (Großbritannien, Australien, Neuseeland, USA) etabliert ist. Im Grundsatz beinhaltet sie die Zuordnung einer medikamentösen Wirkgruppe zu einer bestimmten Farbe (z. B. Opioide hellblau). Der zugrundeliegende Sicherheitsaspekt beruht auf der Annahme, dass Verwechslungen innerhalb einer Medikamentengruppe weniger schwerwiegende Konsequenzen haben. Vor nunmehr 5 Jahren wurde die Umsetzung der ISONorm vom Präsidium der DGAI als Empfehlung verabschiedet.

Vielversprechende Ansätze, um Fehler in der Anästhesie zu vermeiden, sind z. B. die farbliche Kennung von Medikamenten einer Wirkgruppe sowie die Implementierung der Surgical Safety Checklist.

Perioperatives Wärmemanagement Für die perioperative Hypothermie konnte gezeigt werden, dass sie ein erheblicher Risikofaktor für die Entwicklung schwerer postoperativer Komplikationen ist. Am häufigsten assoziiert ist sie mit kardialen Komplikationen, erhöhten Blutverlusten und Transfusionsraten sowie vermehrt postoperativen Wundinfekten [23, 24]. Konsequenterweise reduziert ein optimiertes perioperatives Wärmemanagement diese Komplikationen signifikant, mit dem Resultat eines verbesserten Outcomes der Patienten [25-27].

Zielorientierte intraoperative hämodynamische Optimierung Seit vielen Jahren liegen überzeugende Daten dafür vor, dass eine frühe intraoperative hämodynamische Optimierung die postoperativen Komplikationen, den Intensivaufenthalt und die Krankenhausaufenthaltsdauer signifikant reduzieren kann [28-31]. Die tatsächliche Umsetzung bzw. Implementierung proto- 
kollgestützter Optimierungskonzepte in den klinischen Alltag gestaltet sich allerdings schwierig. In einer Umfrage an verschiedenen Kliniken der Vereinigten Staaten und Europas zum intraoperativen hämodynamischen Monitoring bei nicht herzchirurgischen Patienten wurden klinische Erfahrung, Blutdruckmonitoring, Urinausscheidung und zentraler Venendruck am häufigsten genannt [32]. Auf die Frage, ob das intraoperative Monitoring verbessert werden könnte, antworteten $86,5 \%$ der amerikanischen Kollegen und 98,1\% der europäischen Kollegen mit ,ja“. Grundsätzlich weiß man um die Notwendigkeit eines erweiterten intraoperativen hämodynamischen Monitorings bei Risikopatienten und / oder Risikoeingriffen, bei der Umsetzung gibt es jedoch noch viel Potenzial.

Patient Blood Management Ein weitaus umfassenderes und ambitionierteres Projekt ist das „Patient Blood Management“-Programm, dessen Einführung in den klinischen Alltag die WHO seit 2011 fordert [33-35]. Seit ca. 2 Jahren wird dieses im Kern multidisziplinäre Projekt zur Optimierung des perioperativen Umgangs mit der „Ressource Blut" auch in Deutschland zunehmend umgesetzt [36-38]. Ein Teilaspekt dieses Projekts sei an dieser Stelle nur exemplarisch aufgeführt: die präoperative Anämie.

Der Anteil an Patienten, die sowohl im herzchirurgischen als auch im nicht herzchirurgischen Bereich mit einer Anämie zur operativen Versorgung vorgestellt werden, ist hoch. In einer 2009 von Beattie et al. veröffentlichten Studie wurde bei 39\% der insgesamt 7679 untersuchten nicht herzchirurgischen Patienten eine präoperative Anämie (nach WHO-Definition) festgestellt. Die Patientengruppe mit Anämie zeigte eine deutlich höhere 90-Tage-Mortalität im Vergleich zu den Patienten ohne Anämie [39]. Nun ist jedoch genau dieser Punkt, das Erkennen und Optimieren einer präoperativen Anämie, eine der größten interdisziplinären Herausforderungen im Rahmen des „Patient Blood Management“-Programms. Es wird viel Zeit und Engagement aller behandelnden Disziplinen erfordern, hier im Sinne einer verbesserten Patientensicherheit zu kooperieren.

Mit dem perioperativen Wärmemanagement, der intraoperativen zielorientierten hämodynamischen Optimierung sowie dem Patient Blood Management werden derzeit 3 Behandlungskonzepte zur Reduktion postoperativer Komplikationen breiter in den klinischen Alltag implementiert.

„Critical Incident Reporting System“ (CIRS) Im November 1999 hat das „Institute of Medicine“ mit einer denkwürdigen Ausgabe mit dem Titel „To Err is Human: Building a Safer Health System“ in den Vereinigten Staaten ein Bewusstsein für Fehler in der Medizin geschaffen. Dem Report zufolge versterben in den USA zwischen 44000 und 98000 Patienten aufgrund medizinischer Fehlentscheidungen. Die Autoren betonen, dass Fehler ein unabwendbarer Bestandteil auch des anästhesiologischen Alltags sind. Wenn nun Fehler passieren, sollten diese jedoch auch genutzt werden, um innerhalb eigener, aber auch übergeordneter Strukturen Ursachen zu identifizieren und Lösungen konstruktiv zu erarbeiten, um diese Fehler oder auch Beinahe-Fehler in Zukunft vermeiden zu können [40].

Mit dem CIRSmedical Anästhesiologie (CIRSAINS) haben der BDA, die DGAI und das Ärztliche Zentrum für Qualität in der Medizin (ÄZQ) ein gemeinsames Modellprojekt auf den Weg gebracht ( https://www.cirs-ains.de). Dieses EreignisMeldesystem („Critical Incident Reporting System“) bietet ein Forum für die anonyme Erfassung und Auswertung von Ereignissen und BeinaheEreignissen in der Anästhesie, Intensivmedizin, Notfallmedizin und Schmerztherapie, welche die Patientensicherheit gefährdet haben oder hätten gefährden können.

\section{Perioperative Medizin - in der Hand des Anästhesisten? $\nabla$}

Fokus auf gesamten perioperativen Prozess Die Deklaration von Helsinki zur Patientensicherheit in der Anästhesiologie stellt die große Verantwortung außer Frage, die das Fachgebiet Anästhesiologie schon heute für Qualität und Sicherheit sowohl in der Anästhesie, aber auch in der Intensiv- und Notfallmedizin sowie der Schmerztherapie hat. Darüber hinaus haben sich die führenden Repräsentanten der nationalen anästhesiologischen Fachgesellschaften dazu bekannt, den gesamten perioperativen Prozess sowie die vielen anderen potenziell patientengefährdenden Situationen inner- und außerhalb des Krankenhauses künftig mehr in den Fokus zu stellen und in den anästhesiologischen Alltag zu implementieren.

Was bedeutet das für unsere Fachdisziplin? Wir konnten in den letzten Jahren auf der Basis pharmakologischer und technologischer Innovationen in erster Linie die intraoperative Versorgung sowie die intensivmedizinische Therapie optimieren. Wie sich der weitere postoperative Verlauf unserer Risiko- oder Hochrisikopatienten gestaltet, nachdem sie den Aufwachraum oder die Intensivstation verlassen, wissen wir häufig nicht. In aller Regel spielen wir als Anästhesisten im postoperativen Setting auf der Normalstation eine untergeordnete Rolle.

Grundsätzlich muss das natürlich nicht so sein. Die Anästhesie hat seit vielen Jahren mit dem Akut-Schmerzdienst bewiesen, dass eine postoperative Schmerzvisite das Outcome der Patienten verbessern kann und dass sich Innovationen 
im Sinne der Patientensicherheit flächendeckend und nachhaltig etablieren und weiterentwickeln können [41, 42].

\section{„It happens on the ward“}

Goldhill et al. Schon 1999 analysierten Goldhill und Mitarbeiter das Management und Outcome von Patienten, die von einer Normal- auf die Intensivstation aufgenommen wurden und in den letzten $24 \mathrm{~h}$ vor Übernahme nicht operiert worden waren [43]. Ein Drittel der identifizierten Patienten wurde unmittelbar vor Übernahme reanimiert, die Mehrzahl von ihnen war vorher sauerstoffpflichtig, die Sauerstoffsättigung wurde gemessen und eine arterielle Blutgasanalyse schon auf Normalstation angewiesen. Zwei Drittel dieser Patienten zeigte eine Sauerstoffsättigung $<90 \%$. Die Krankenhausmortalität dieser Patientengruppe lag bei $58 \%$.

Story et al. Einige Jahre später wurden diese Daten durch eine Untersuchung von Story und Kollegen untermauert [44]. Diese Arbeitsgruppe konnte zeigen, dass durch die Kombination einer postoperativen Schmerzvisite mit einer „Critical Care Outreach"-Visite die postoperative Morbidität und Mortalität signifikant reduziert werden konnte. Ernsthafte, schwere Zwischenfälle konnten von 23 auf 16 pro 100 Patienten reduziert werden, assoziiert mit einer Abnahme der 30 -Tage-Mortalität von $9 \%$ auf $3 \%$.

Findlay et al. Eine jüngere Untersuchung aus dem Jahr 2011 von Findlay et al. zur perioperativen Sicherheit chirurgischer Patienten identifizierte folgende Probleme [45]:

- mangelhafte präoperative Risikoeinschätzung

- fehlende intraoperative Monitoring-Protokolle

- mangelhafte individualisierte postoperative Überwachung

Nur 22\% der Patienten mit hohem Risiko wurden postoperativ auf der Intensivstation überwacht; $48 \%$ der Hochrisikopatienten, die verstarben, waren nie auf einer Intensivstation [45].

EuSOS-Studie Aktuelle Daten aus der kürzlich veröffentlichten EuSOS-Studie haben diese Ergebnisse untermauert und die Notwendigkeit zu reagieren noch deutlicher gemacht: $70 \%$ der Patienten aus der EuSOS-Studie, die im Krankenhaus verstarben, wurden nie auf eine Intensivstation übernommen [46].

Fragen für die Zukunft Zusammenfassend zeigen die hier aufgeführten Untersuchungen wenigstens 3 Probleme:

1. Eine postoperative Verschlechterung unserer Patienten auf der Normalstation wird z.T. schon dokumentiert, offensichtlich jedoch mangelhaft wahrgenommen und/oder kommuniziert.
2. Die erforderlichen Interventionen werden in der Folge zu spät initiiert.

3. Wir haben offensichtlich ein Problem mit der entsprechenden Planung und Vorhaltung von Intensivkapazitäten für die Patienten, die eine Intensivtherapie benötigen.

Zwangsläufig ergeben sich hieraus Fragen, mit denen wir uns künftig dringend auseinandersetzen müssen, um Strategien zu entwickeln, die o.g. Probleme zu minimieren:

- Wie können wir die Allokation von Intensivbetten optimieren?

- Wie können wir eine adäquate postoperative Überwachung von Risikopatienten auf der Normalstation implementieren?

- Brauchen wir darüber hinaus individualisierte hämodynamische Optimierungsprotokolle bei spontan atmenden Patienten auf der Normalstation?

Um die Patientensicherheit in der Anästhesiologie perioperativ zu verbessern, bieten alle 3 Teilbereiche weiteres Optimierungspotenzial:

- Verbesserung der präoperativen Gesamtrisikoeinschätzung von Patient und geplantem Eingriff,

- Implementierung intraoperativer Behandlungsalgorithmen und

> individualisierte postoperative Überwachungskonzepte bei Risikopatienten.

\section{Resümee und Ausblick}

Aktueller Stand Grundsätzlich verfügen wir derzeit über eine hinreichende Evidenz zum Thema Patientensicherheit und Outcome in der Anästhesie. Die kritischen Bereiche mit Optimierungspotenzial sind identifiziert und zunehmend Gegenstand internationaler, vielversprechender Initiativen in der Anästhesie. Als Reaktion auf die Deklaration von Helsinki zur Patientensicherheit in der Anästhesiologie wird jetzt regelhaft im Rahmen des jährlichen Kongresses der Europäischen Gesellschaft für Anästhesiologie (ESA) der „European Patient Safety Course“ angeboten: ein 8-stündiger Kurs aus Vorträgen, Fallbesprechungen und Workshops.

Europäische CIRS-Plattform Darüber hinaus sollen künftig die „Critical Incident Reporting“Systeme durch die Etablierung einer europäischen Plattform, der „Anaesthesia Safety Alert Platform“ (ASAP), weiter gefördert werden. Auf dieser Plattform sollen europaweit Sicherheitswarnungen und Empfehlungen aus den verschiedenen europäischen „Critical Incident Reporting“Systemen gesammelt und zur Verfügung gestellt werden. 


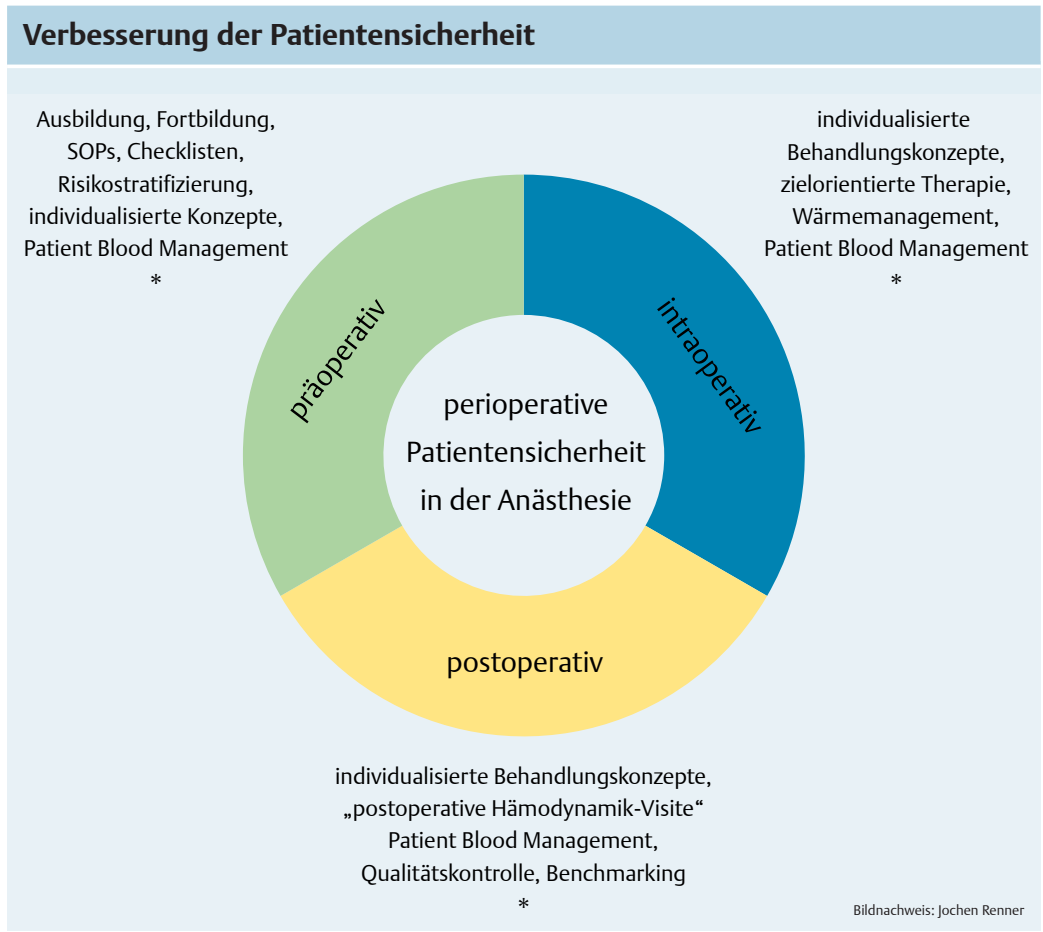

Abb. 4 Etablierte Konzepte und im Prozess befindliche Ansätze, die Patientensicherheit in der Anästhesie weiter zu verbessern (*ohne Anspruch auf Vollständigkeit).
Ausdehnung auf perioperativen Bereich Für die Zukunft wäre es wünschenswert, den Aspekt Patientensicherheit in der Anästhesie über den unmittelbaren intraoperativen Bereich hinaus zu erweitern und zumindest bei Risikopatienten auf den gesamten perioperativen Aufenthalt auszudehnen. So lassen sich in der Summe einige Punkte zusammentragen, die zusätzlich zu Checklisten, protokollbasierten Behandlungskonzepten und „Critical Incident Reporting“Systemen helfen werden, das Outcome unserer Patienten zu verbessern ( $\bullet$ Abb. 4). Mit der Kenntnis um diese Notwendigkeit, mit einer ständigen medialen Präsenz der Thematik und mit entsprechenden Ressourcen werden weiterhin Verbesserungen in der anästhesiologischen Patientenversorgung möglich sein. Ziel muss es sein, diese Thematik zu einem integralen Bestandteil unseres täglichen Handelns werden zu lassen - dann gibt es keinen Zweifel an der Antwort auf die Frage: „Kann der Anästhesist das Outcome verbessern?"

Beitrag online zu finden unter http://dx.doi. org/10.1055/s-0040-100222
Fazit Grundsätzlich verfügen wir derzeit über eine hinreichende Evidenz zum Thema Patientensicherheit und Outcome in der Anästhesie und es gibt bereits eine Reihe vielversprechender, weitgehend „evidenzbasierter“ Konzepte in der Anästhesiologie, die nachweislich die Patientensicherheit verbessern können. Einige dieser Konzepte sind z.T. schon in den klinischen Alltag implementiert oder finden zunehmend Akzeptanz und Verbreitung. Für die Zukunft wäre es wünschenswert, den Aspekt „Patientensicherheit in der Anästhesie“ über den unmittelbaren intraoperativen Bereich hinaus zu erweitern und zumindest bei Risikopatienten stärker auf den gesamten perioperativen Aufenthalt auszudehnen. Mit dem Wissen um diese Notwendigkeit, der unablässlichen Sensibilisierung für das Thema und der Vorhaltung entsprechender Ressourcen werden weiterhin Verbesserungen in der anästhesiologischen Patientenversorgung möglich sein.

Kernaussagen

Es gibt eine Reihe vielversprechender, weitgehend „evidenzbasierter“ Konzepte in der Anästhesiologie, welche die Patientensicherheit verbessern können und die z. T. bereits in den klinischen Alltag implementiert sind.

Zu diesen Konzepten gehören u. a.: die „Surgical Safety Checklist", die einheitliche farbliche Kennzeichnung von Medikamenten einer Wirkgruppe, das perioperative Wärmemanagement, die zielorientierte intraoperative hämodynamische Optimierung, das Patient Blood Management und "Critical Incident Reporting"-Systeme.

Künftig sollte der Aspekt „Patientensicherheit in der Anästhesie“ über den unmittelbaren intraoperativen Bereich hinaus erweitert und zumindest bei Risikopatienten stärker auf den gesamten perioperativen Aufenthalt ausgedehnt werden.

Potenzial zur Verbesserung der Patientensicherheit in der Anästhesie bieten hierfür alle 3 Teilbereiche:

Verbesserung der präoperativen Gesamtrisikoeinschätzung von Patient und geplantem Eingriff

Implementierung intraoperativer Behandlungsalgorithmen

individualisierte postoperative Überwachungskonzepte bei Risikopatienten 


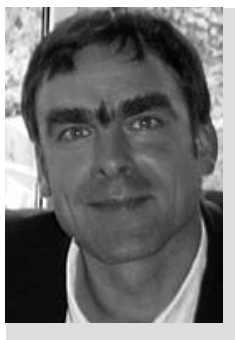

PD Dr. med. Jochen Renner ist Ltd. Oberarzt und Lehrkoordinator der Klinik für Anästhesiologie und Operative Intensivtherapie, Universitätsklinikum Schleswig-Holstein, Campus Kiel. Seine Schwerpunkte sind: hämodynamisches Monitoring,

zielorientierte hämodynamische

Optimierung, Kardioanästhesie

(Erwachsene und Pädiatrie).

E-Mail: jochen.renner@uksh.de

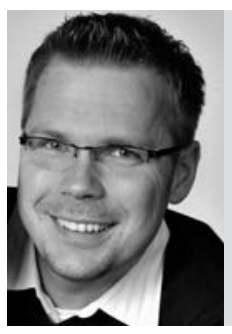

PD Dr. med. Matthias Grünewald ist Oberarzt der Klinik für Anästhesiologie und Operative Intensivtherapie, Universitätsklinikum Schleswig-Holstein, Campus Kiel. Sein Schwerpunkt ist hämodynamisches Monitoring. E-Mail: matthias.gruenewald@uksh.de

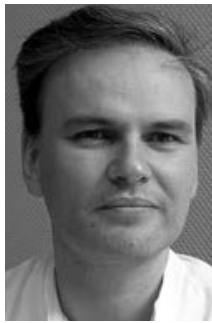

Prof. Dr. med. Berthold Bein, MA, DEAA, ist Chefarzt der Abteilung für Anästhesiologie und Intensivmedizin, Asklepios Klinik St. Georg Hamburg. Seine Schwerpunkte sind: hämodynamisches Monitoring. Kardioprotektion, Patient Blood Management.

E-Mail: b.bein@asklepios.com

Interessenkonflikt Die Autoren erklären, dass keine Interessenkonflikte vorliegen.

\section{Literaturverzeichnis}

1 Mellin-Olsen J, Staender S, Whitaker DK, Smith AF. The Helsinki Declaration on Patient Safety in Anaesthesiology. Eur J Anaesthiol 2010; 27: 592-597

2 Hayes JA, Black NA, Jenkinson C et al. Outcome measures for adult critical care: a systematic review. Health Technol Assess 2000; 4: 1-111

3 Brandt L, Krauskopf KH. "A discovery in surgery". 150 years of anesthesia. Anaesthesist 1996; 45: 970-975

4 Goerig M, Schulte am Esch J. Helmut Schmidt - Ein Protagonist moderner Anästhesie in Deutschland. Anasthesiol Intensivmed Notfallmed Schmerzther 1996; 31: 621-631

5 Macintosh RR. Deaths under anaesthetics. Br J Anaesth 1949; 21: 107-136

6 Schiff JH, Welker A, Fohr B et al. Major incidents and complications in otherwise healthy patients undergoing elective procedures: results based on 1.37 million anaesthetic procedures. Br J Anaesth 2014; 113: 109-121

7 Arbous MS, Grobbee DE, van Kleef JW et al. Mortality associated with anaesthesia: a qualitative analysis to identify risk factors. Anaesthesia 2001; 56: 1141-1153

8 Braz LG, Braz DG, Cruz DS et al. Mortality in anesthesia: a systematic review. Clinics 2009; 64: 999-1006

9 Duncan PG. The image of the anaesthetist. Eur J Anaesthesiol Suppl. 1993; 7: 3-7

10 Lienhart A, Auroy Y, Pequignot F et al. Survey of anesthesiarelated mortality in France. Anesthesiology 2006; 105: 1087-1097

11 Eichhorn JH. Prevention of intraoperative anesthesia accidents and related severe injury through safety monitoring. Anesthesiology 1989; 70: 572-577

12 Li G, Warner M, Lang BH et al. Epidemiology of anesthesiarelated mortality in the United States, 1999-2005. Anesthesiology 2009; 110: 759-765

\section{Literatur online}

Das vollständige Literaturverzeichnis zu diesem Beitrag finden Sie im Internet:

\section{Abonnenten und Nicht-}

abonnenten können unter "www.thieme-connect.de/ ejournals “ die Seite der AINS aufrufen und beim jeweiligen Artikel auf „Zusatzmaterial“ klicken - hier ist die Literatur für alle frei zugänglich. 